\title{
Evaluación de los Diferentes Niveles de Diatomea en la Fase de Crecimiento - Engorde de Cavia Porcellus (Cuyes)
}

\author{
Luis Condolo, Master en Farmacología \\ Willam Maurat, Ingeniero Zootecnista \\ Antonio Velasco, Magister en Gestion de Proyectos Socio \\ Productivos \\ Diego Cajamarca, Magister en Sistemas Integrados de Gestion \\ de la Calidad Ambiente y Seguridad \\ Maritza Vaca, Magister en Cadenas Productivas \\ Agroindustriales \\ Escuela Superior Politécnica de Chimborazo, ESPOCH, Ecuador
}

Doi: 10.19044/esj.2019.v15n18p131 URL:http://dx.doi.org/10.19044/esj.2019.v15n18p131

\section{Resumen}

En la provincia de Morona Santiago, Cantón Sucúa, la crianza de conejillos de indias se realiza de forma empírica con bajos rendimientos. Por lo que se evaluaron tres niveles de diatomeas para controlar el tratamiento del peso en crecimiento y la carga parasitaria $(1,5,3,0$ y 4,5\%) (T0 adicional) en 48 cuyes de la línea peruana mejorada, de 15 días de edad y un peso promedio de $375.96 \mathrm{~g}$, que se distribuyeron bajo un Diseño Completamente Aleatorio (DCA), que consta de 6 repeticiones, y un tamaño de unidad experimental de 2 cobayas por repetición. Los datos se aplicaron al análisis de varianza, separación de significados según Tukey $(0.05$ y 0.01) y análisis de regresión y correlación. En relación a los resultados, el tratamiento T2 (3\% de diatomeas) alcanzó un peso final de $964.50 \mathrm{~g}$ y un aumento en la ganancia de peso de $624.08 \mathrm{~g}$; la conversión alimenticia más eficiente de 6.66, el producto alimenticio total más bajo con el T1 (1.5\%) de 4113.05 g MS. Se determinó que con el tratamiento T3 (4.5\%), la carga parasitaria de $56.86 \%$ que estaba al comienzo del experimento se redujo a $4.90 \%$ después de 90 días. La rentabilidad más alta fue el tratamiento T2 (3\% diatomea) con un beneficio / costo de 1.19 , lo que significa que por cada dólar invertido hay un rendimiento de 0.19 dólares (USD). Se sugiere entonces, aplicar en la etapa de crecimiento: engordar el 3\% de las diatomeas en las dietas para conejillos de indias. Esta dosis resulta ser la mejor cuando se consideran los parámetros productivos y económicos. 
Palabras clave: Diatomeas, Cuyes, Crecimiento, Engorde, Comportamiento productivo

\title{
Evaluation of Different Levels of Diatomea in the Phase of Growth - Fattening in Cavia Porcellus (Cuyes)
}

\author{
Luis Condolo, Master en Farmacología \\ Willam Maurat, Ingeniero Zootecnista \\ Antonio Velasco, Magister en Gestion de Proyectos Socio \\ Productivos \\ Diego Cajamarca, Magister en Sistemas Integrados de Gestion \\ de la Calidad Ambiente y Seguridad \\ Maritza Vaca, Magister en Cadenas Productivas \\ Agroindustriales
}

Escuela Superior Politécnica de Chimborazo, ESPOCH, Ecuador

\begin{abstract}
In the province of Morona Santiago, Cantón Sucúa, the breeding of guinea pigs is done empirically with low yields. Therefore, three levels of diatoms were evaluated to control the treatment of growing weight and parasitic load (1.5, 3.0 and 4.5\%) (additional T0) in 48 guinea pigs of the improved Peruvian line of 15 days of age and an average weight of $375.96 \mathrm{~g}$. These were distributed under a Completely Random Design (CRD), which consists of a frequency of 6 , and an experimental unit size of 2 guinea pigs per frequency. The data were applied to the analysis of variance, separation of meanings according to Tukey (0.05 and 0.01) and regression and correlation analysis. In relation to the results, the treatment $\mathrm{T} 2$ (3\% of diatoms) reached a final weight of $964.50 \mathrm{~g}$ and an increase in the weight gain of $624.08 \mathrm{~g}$. Also, the most efficient feed conversion is 6.66, while the lowest total food product with the T1 (1.5\%) is $4113.05 \mathrm{~g}$ MS. It was determined that with the T3 treatment (4.5\%), the parasitic load of $56.86 \%$ that was at the beginning of the experiment was reduced to $4.90 \%$ after 90 days. The highest profitability was the T2 treatment (3\% diatom) with a benefit / cost of 1.19. This means that for every dollar invested, there is a yield of 0.19 dollars (USD). It was suggested then the growth stage should be applied i.e. 3\%
\end{abstract}


fattening of the diatoms in the diets for guinea pigs. This dose is the best when considering the productive and economic parameters.

Keywords: Diatoms, Guinea pigs, Growth, Fattening, Productive behavior

\section{Introduction}

La producción de cuyes en el Ecuador ha llegado a considerarse una alternativa de alimentación humana que brinda carne de calidad con un alto contenido nutricional, en el país se estima que existe una población de 5 067.049 de cobayos, la crianza y explotación en la actualidad ha traspasado las fronteras y es demandado en los mercados de América del Norte, Unión Europea y Asía, por contener carne de proteína alta, grasa baja, Omega 3, hierro, vitaminas A, B1, B2, B6, B12, C, D, E, K, ácido fólico, y minerales como el calcio, fósforo, magnesio, manganeso y potasio, que resulta atractiva para el consumo humano.

La alimentación y nutrición es uno de los factores determinantes en la producción de cuyes, los costos elevados, escases de materia prima y aditivos nos lleva a pensar en nuevas alternativas de elaboración de dietas nutricionales para ésta especie animal.

A pesar de ser el cuy un animal rustico y prolífico, ha sido afectado por problemas sanitarios principalmente de tipo parasitario que ha ocasionado retardo en el crecimiento y bajas ganancias de peso, afectando al productor con pérdidas económicas; por lo cual la presente investigación proporciona una alternativa de solución con la administración de diatomeas en la nutrición de los cuyes.

Las diatomeas, al ser algas unicelulares microscópicas, poseen propiedades insecticidas, antibacterianas y antihelmíntico natural, juega un papel importante en el manejo nutricional del cuy, mejorando así las características organolépticas de manera orgánica, además coadyuva con la creación de anticuerpos en el cuy previniendo enfermedades, de esta manera mejora la sanidad animal y contribuye con el equilibrio ecológico al mitigar las emisiones de olor en sus excretas.

En Ecuador existen familias con escasos recursos económicos, dedicados a la agricultura tradicional, cuya producción no supera el consumo familiar y no generan excedentes que mejoren la calidad de vida de la población. Éstos núcleos familiares se dedican a la crianza de especies menores de forma tradicional dando lugar a bajos rendimientos productivos y reproductivos del animal. La investigación da a conocer un sistema alternativo de alimentación que genere un mayor rendimiento productivo durante la crianza y que permita al productor reducir sus costos de producción. 
En la presente investigación se planteo la utilización de diferentes niveles de diatomea $(1,5 ; 3$ y 4,5 \%), en la suplementación alimenticia de cuyes en la fase de crecimiento - engorde, Además de determinar el comportamiento productivo de los cuyes bajo el efecto de diferentes niveles de diatomea, como una alternativa de suplemento alimenticio y establecer la rentabilidad económica de los tratamientos a través del indicador beneficio costo $(\mathrm{B} / \mathrm{C})$.

\section{Materiales y Metodos}

La investigación se realizó en el cantón Sucua, provincia de Morona Santiago, a una temperatura de $22,3{ }^{\circ} \mathrm{C}$ y a una altura de $850 \mathrm{msnm}$. Se utilizo 48 cuyes mejorados de 15 días de edad y un peso promedio de 375,96 g. Con tres tratamientos a base de diferentes niveles de diatomitas $(1,5 ; 3,0 \mathrm{y}$ $4,5 \%$ ) para su comparación con un tratamiento testigo (T0). Se aplicó un diseño Completamente al Azar, conformado por 6 repeticiones, un tamaño de unidad experimental de 2 cuyes por repetición, 12 cuyes por tratamiento.

Los resultados obtenidos fueron sometidos a los siguientes análisis estadísticos: Análisis de varianza (ADEVA); Separación de medias según Tukey a un nivel de significancia de $\mathrm{P} \leq 0,05$ y $\mathrm{P} \leq 0,01$ y Análisis de regresión. Los animales se alojaron en jaulas de 0,5 x 0,5 x 0,4 metros; se colocaron 2 animales por jaula las mismas que disponían de un comedero y un bebedero. Los pesos se tomaron cada 30 días de edad, iniciando con el peso al destete, hasta el peso final a los 105 días de edad. Al terminar el experimento (90 días), fueron pesados por última vez para determinar su peso al sacrificio. Además se revisó diariamente cada una de las pozas, para verificar anomalías en los animales.

Las variables de respuestas evaluadas fueron peso inicial y final $(\mathrm{g})$, conversión alimenticia $(\mathrm{g})$.

El alimento se suministró de acuerdo a los requerimientos establecidos para las etapas de crecimiento - engorde que consistió en: administrar $200 \mathrm{~g}$ de forraje verde de mar-alfalfa el cual tiene la siguiente composición nutricional (Humedad 70,33\%; Cenizas 13,50\%; Fibra 53,33\%; Grasa 2,10\%; Carbohidratos solubles 12,20\%; Proteínas crudas 16,25\%; Calcio 0,80\%; Fósforo 0,33\%) en el alimento concentrado se añadió los siguientes niveles de diatomeas descritos en el cuadro 1 .

Cuadro 1. Consumo de Concentrado más Adición de Diatomea \%.

\begin{tabular}{lrrrrr}
\hline \multirow{2}{*}{$\begin{array}{c}\text { Periodo de } \\
\text { investigación }\end{array}$} & $\begin{array}{c}\text { Consumo de } \\
\text { forraje verde, } \mathrm{g}\end{array}$ & Consumo de & \multicolumn{3}{c}{ Adicción de diatomeas, $\mathrm{c}$} \\
\cline { 5 - 7 } 1 a 30 días & 80 & 15 & 0,23 & 0,45 & 0,68 \\
31 a 60 días & 134 & 20 & 0,3 & 0,6 & 0,9 \\
61 a 90 días & 220 & 30 & 0,45 & 0,9 & 1,35 \\
\hline
\end{tabular}


El experimento se inició con la limpieza y desinfección de las pozas, instalaciones y equipos, para lo cual se utilizó amonio cuaternario en una proporción de $20 \mathrm{ml} / 10$ lts de agua. La desinfección se realizó tres veces durante toda la investigación, como parte de sus raciones diarias se suministró agua Ad libitum conjuntamente con el alimento concentrado y forraje verde el cual se registró cada día, además considerando sus residuos para determinar el consumo diario de materia seca. Para el análisis coproparasitario se utilizó el método de flotación para identificar la presencia de huevos y ovocitos de parásitos gastrointestinales como : Unicaria, Entamoeba coli, Trichuris trichiura, etc.

\section{Resultados y Discusión}

La variable de los cuyes fueron con pesos iniciales homogéneos de 401,$17 ; 383,58 ; 378,67$ y 340,42 g para los tratamientos con la aplicación del T0; 1,$5 ; 3,0$ y $4,5 \%$ de diatomeas respectivamente, permitiendo que el trabajo experimental no se incline a ninguno de los tratamientos establecidos. En su investigación se emplearon diferentes niveles de Genex como promotor de crecimiento inicial con un peso promedio de $375,67 \mathrm{~g}$, datos similares con la presente investigación, además de conocer que los animales se destetan a los 14 días con peso promedios de $350 \mathrm{~g}$ (Mayorga, 2015).

Con respecto al peso final, no se registraron diferencias estadísticamente significativas $(\mathrm{P}>0,05)$ pero si diferencias descriptivas; el mayor valor registró el tratamiento T2 $(3,0 \%)$ con $964,5 \mathrm{~g}$ y el de menor valor el tratamiento T3 (4,5\%) con 932,17 g. Estas diferencias se deben a la administración de diatomeas en diferentes porcentajes en la alimentación, cumplimiento como excelente suplemento nutritivo mineral con altos contenidos de calcio, sílice y oligoelementos, que lo hacen importante en la nutrición animal (Illana, 2002). Al emplear diferentes niveles de harina de hoja de yuca en la alimentación diaria de los cuyes en la etapa de crecimiento engorde, registró un peso final de 805,39 gramos con la aplicación del $45 \%$ de mencionada harina (Pasquel, 2010). Al usar en la dieta de los cuyes diferentes niveles de clorhidrato de ractopamina registró un peso promedio de 926,87 gramos, valor que resulta ser inferior al registrado en la presente investigación, lo cual podría deberse a que las diatomea tienen un mayor contenido de nutrientes con respecto al clorhidrato de ractopanin (León, 2015).

Según la ganancia de peso mostró diferencias estadísticas altamente significativas $(\mathrm{P}<0,01)$, registrándose entre las unidades experimentales la mayor ganancia de peso en animales del tratamiento con el 3,0\% de diatomea, con una media de 624,08 g; seguido por los cuyes con la adición de 1,5\% de diatomea, con $575,42 \mathrm{~g}$; y posteriormente se alcanzaron los menores pesos finales de 548,58 y 535,67 g, en las unidades experimentales del tratamiento con el uso del 4,5 y $0 \%$ de diatomea en la alimentación diaria, 
respectivamente. Arthur (2000) reporta que las diatomeas poseen en su composición minerales y sustancias que ayudan en la prevención y eliminación de agentes patógenos entre estos la E. coli; y refuerza el sistema inmunológico gracias al crecimiento de la microflora intestinal lo cual hace que el animal por su buen estado de salud mejore considerablemente sus condiciones productivas, dando mayor rentabilidad al productor.

El consumo de forraje verde en gramos de materia seca registró diferencias estadísticas $(\mathrm{P}<0,01)$, por efecto de la aplicación de los diferentes niveles de diatomea, los mayores valores de consumo presentaron los tratamientos T0 con 2265,12 y T2 2229,88 gramos de materia seca (g Ms), los menores consumos de forraje verde fue de 2216,62 y 2184,71 gMS para los tratamientos $\mathrm{T} 3$ y $\mathrm{T} 1$ respectivamente, evidenciando que el empleo de diferentes niveles de diatomea, disminuyen el consumo de forraje en los semovientes quizás esto se deba a que los animales mejoran su digestibilidad por la adicción de la diatomea.

El consumo de concentrado registró diferencias estadísticas altamente significativas $(\mathrm{P}<0,01)$ entre los tratamientos, el mayor consumo presentó el tratamiento T0 (0\%) con un valor de $1934,51 \mathrm{gMs}$, y el menor valor el tratamiento T3(4,5\%) con 1905,41 gramos; los demás tratamientos registraron valores intermedios a los mencionados anteriormente; posiblemente éstas diferencias se deban al efecto de la palatabilidad al incluir los diferentes niveles de diatomeas en la dieta de los cuyes.

León (2015), al emplear varios niveles de clorohidrato de ractopamina, alcanzó un consumo de concentrado de $4,56 \mathrm{~kg}$ en la evaluación a los 90 días. Padilla (2012), al emplear diferentes niveles de orégano en la dieta de los cuyes alcanzó un consumo de $2,34 \mathrm{~kg}$ de alimento en materia seca, datos que suelen ser mayores a los de la presente investigación, posiblemente esto se deba a que el orégano y el clorhidrato de ractopamina tienen mayor palatabilidad en comparación con las diatomeas.

El consumo total de alimento en gMS se determinó diferencias estadísticas altamente significativas $(\mathrm{P}<0,01)$, el mayor consumo de alimento total fue en el tratamiento control $(0 \%)$ con 4199,63 gramos de materia seca, y el menor consumo registro el T1 $(1,5 \%)$ con 4113,05 gMS, mientras que los demás tratamientos obtuvieron valores intermedios a los mencionados, lo que demuestra que los niveles de diatomeas influyen directamente en la alimentación de los cobayos.

El índice de Conversión alimenticia bajo la separación de medias según Tukey, se reportan diferencias estadísticas altamente significativas $(\mathrm{P}<$ $0,01)$, por efecto de los diferentes niveles de diatomea; la conversión alimenticia más eficiente fue de 6,66 en el T2 (3\% de diatomea); mientras que el tratamiento control (T0\%), obtuvo una conversión menos eficiente de 7,18. Avendaño (2007) menciona que las diatomeas se han utilizado en 
tratamientos de parasitosis, desórdenes gastrointestinales, debido a su efecto cicatrizante y multiplicador de microflora intestinal, coadyuvando con la obtención de mejores rendimientos como pesos finales y por ende ganancia de pesos. León (2015), al emplear varios niveles de clorohidrato de ractopamina, alcanzo un consumo de 6,62 kg en la evaluación a los 90 días; Padilla (2012), al emplear diferentes niveles de orégano en la dieta de los cuyes alcanzó un consumo de $4,52 \mathrm{~kg}$ de alimento en MS, notándose un consumo mayor en comparación con la presente investigación, posiblemente esto se deba a que las diatomeas al ser secuestrante de micotoxinas eleva su digestibilidad lo que hace que el animal consuma menos pero asimile en mayor porcentaje.

De esta manera se determina que el $3 \%$ de diatomea influye positivamente en la conversión alimenticia de los animales, al respecto, Vargas (2012) menciona que la diatomea es un poderoso nutriente que aporta oligoelementos que mejoran la salud de quienes lo consumen, además de ser económico y beneficia el levante y engorde de cualquier especie animal, sin representar un peligro para quienes lo manipulan, ni para los animales que lo consumen.

Chillagano (2014), al incluir el 15\% de amaranto en el balanceado para cuyes en la etapa de crecimiento - engorde, alcanzó una conversión alimenticia de 7,10. León (2015), al emplear diferentes niveles de clorhidrato obtiene en el T2 la menor conversión alimenticia de 10,33; siendo respuestas menos eficientes con respecto a los de la presente investigación, quizás esto se vea influenciado por aspectos climáticos y genéticos de los cobayos.

Moncayo (2015), al emplear diferentes niveles de Genex determina una conversión alimenticia de 3,05, datos más eficientes al contrastar con los de la presente investigación, quizás esto se deba a lo indicado por OPTIVITE (2014), que GENEX promueve la regulación digestiva dentro de las posibilidades de un producto elaborado con ingredientes totalmente naturales, y sus cualidades son mucho más amplias.

Según la infestación parasitaria inicial se considera que al inicio de la investigación no reportaron diferencias estadísticas significativas $(\mathrm{P}>0,05)$, entre los tratamientos evaluados, llegando a tener los menores porcentajes de infestación con la utilización del tratamiento testigo T0 y el del tratamiento (T1) 1,5\% de diatomea con una media de 40,20\% y 36,27 \% respectivamente; los mayores valores fueron de $44,12 \%$ y $56,86 \%$ para los tratamientos $\mathrm{T} 2(3 \%)$ y $\mathrm{T} 3(4,5 \%)$ en su orden.

Según la infestación parasitaria al final de la investigación en los 90 días establecidos, se reportan diferencias estadísticas altamente significativas $(\mathrm{P}<0,01)$, por efecto de la inclusión de los niveles de diatomea en el alimento concentrado, logrando el mayor control de parásitos con los tratamientos $\mathrm{T} 1(1,5 \%), \mathrm{T} 2(3 \%)$ у $\mathrm{T} 3(4,5 \%)$, con cargas parasitarias de $9,81 \% ; 8,83 \%$ y $4,90 \%$ respectivamente, infiriendo de manera significativa con respecto al 
tratamiento control que al finalizar la investigación su carga parasitaria fue de $47,08 \%$.

El análisis realizado se obtuvo que la diatomea tiene propiedades desparasitantes, al incluirlo en la alimentación animal en este caso de los cobayos en la etapa de crecimiento - engorde, que concuerda con lo sugerido por http//. www.Redganadera.Com. (2014), que indica que la tierra de diatomeas "La Tierra Blanca" es inofensiva para el sistema digestivo y aplicándolos en el agua o alimento de consumo diario de los animales, se logrará eliminar parásitos internos como los nemátodos, los cestodos y trematodos. En el campo de la nutrición animal, la tierra de diatomeas está encontrando una rápida aceptación. Sus Beneficios han sido notables en alimentación de rumiantes y monogástricos. Controla diarreas provocadas por procesos bacterianos, es un agente secuestrante de las toxinas bacterianas y actúa como desparasitante. Las diatomeas capturan la toxina antes que ésta se adhiera y provoque daños a las vellosidades.

\section{Conclusion}

La utilización del 3\% de diatomea (T2), en la etapa de crecimiento engorde en los cobayos, alcanzó un peso final de 964,50 gramos, un incremento en ganancia de peso de 624,08 g; la más eficiente conversión alimenticia de 6,66, superando al resto de tratamientos evaluados.

Al evaluar la cuantificación parasitaria en los cuyes alimentados con dietas incluyendo los diferentes niveles de diatomea, con el T3 4,5\% se reduce la carga parasitaria de la infestación inicial que fue del 56,86 \% a un mínimo de infestación parasitaria transcurrido los 90 días, de 4,90\%, corroborando en forma visual, con el bienestar y salud de los animales.

La mayor rentabilidad en la etapa de crecimiento - engorde para los cuyes, se consiguió con el empleo del $3 \%$ de diatomeas, alcanzando un beneficio/costo de 1,19 lo cual significa que por cada dólar invertido existe una rentabilidad neta del $19 \%$.

\section{References:}

1. Affan, A., Karawita, R., Jeon, Y.J. \& Lee, J.B. (2007). Growth characteristics and antioxidant properties of the benthic diatom Navicula incerta (Bacillariophyceae), from Jeju island, Korea. J. Phycol. 43: 823 - 833 .

2. Barrie, A. (2004). Estudio de Mercado: Oferta, Demanda y Comercialización de la Carne de Cuy en la Ciudad de Huancayo, Departamento de Junín; Instituto Ecológico para el Desarrollo; LimaPerú.

3. Batllori, L. (1991). Plantas medicinales y drogas vegetales. Orégano. Offarm 1: p. 80. 
4. Chauca, J. (2014). Conferencias sobre cuyes: IV Symposium de especies animales subutilizadas, Barinas - Venezuela.

5. Chicaiza, D. (2014). Caracterización de la producción de cuyes para la comercialización asociativa en la asociación "Pakusumi" de la parroquia Pasa de la provincia de Tungurahua, Universidad Estatal de Bolívar, Guaranda.

6. Organización de Las Naciones Unidas Para la Agricultura y la Alimentación (2009). Animal Feed Resources Information System, Blood, revisadoen:http://www.fao.org/ag/AGA/AGAP/FRG/AFRIS/Data/3 17.htm. 18/11/2014.

7. Flores, D. (2016). Producción de cuyes en los cantones de la provincia de Chimborazo para su comercialización y exportación a lospaísesdeEspañaeItalia.Disponibleen:http://www.slideshare.net/be navides1969/tesis-carne-de-Cuy.

8. Morgan, O. (1998). Metodología de Investigación, cultivo de cacao Mc Graw Hill Interamericana Editores S.A. Segunda Edición, México

9. Navarrete, G. (2010). Efecto de la cascara de cacao en sustitución de la alfalfa, en la alimentación de conejos mejorados, Lima - Perú. p.7887.

10. Ordoñez, P. (2007). Investigación realizada en cuyes, nutrición, selección y mejoramiento en el Perú 1a ed. Nariño, Colombia Edit. Universidad de Nariño. P 75.

11. Orozco, V. (2015). Ventajas del uso de la Ractopamina. Disponible en https://lapisa.wordpress.com/2015/09/04/ventajas-del-uso-de-laractopamina-en-cerdos/ 